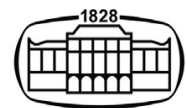

AKADÉMIAI KIADÓ

Journal of Behavioral Addictions

9 (2020) 3, 606-616

D01:

$10.1556 / 2006.2020 .00070$

(c) 2020 The Author(s)

\section{FULL-LENGTH REPORT}

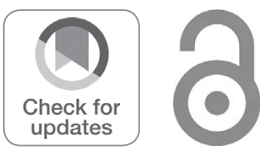

\title{
The relationship between the skill-challenge balance, game expertise, flow and the urge to keep playing complex mobile games
}

\author{
CHANEL J. LARCHE* $\odot$ and MIKE J. DIXON
}

University of Waterloo, 200 University Ave. West, Waterloo, ON, N2L 3G1, Canada

Received: April 10, 2020 • Revised manuscript received: August 04, 2020 • Accepted: September 11, 2020

Published online: October 6, 2020

\begin{abstract}
Background and aims: Interest surrounding the relationship between flow and problematic gameplay has surged. An important antecedent of flow in the context of video-gaming is the skill-challenge balance, but researchers have only manipulated this balance by changing speed of play. The current research seeks to examine the skill-challenge balance and flow in a mobile game in which challenge is increased via the complexity of puzzles. We predicted games like Candy-Crush would more strongly support a model of flow in which the greatest flow would be experienced by more skilled players and that high flow games would induce the most urge to continue play. Methods: We had 60 Candy-Crush players play games near their level standing (maximal skill-challenge balance), or games that were too easy or too hard. Perceived skill, challenge, flow, and urge to continue gameplay were measured after each game. Results: Players felt the highest degree of skill-challenge balance when playing games around their level standing. Easy games produced the least flow, while both regular and hard games produced comparable flow despite hard games being far more challenging and frustrating. The findings support models of flow positing those with highest perceived skill will experience greater flow. Finally, flow and arousal combine to increase urge to keep playing. Discussion and conclusions: Our findings suggest those with high perceived skill will experience deep, immersive flow which motivates players to keep playing.
\end{abstract}

\section{KEYWORDS}

flow, skill-challenge balance, gaming, mobile game addiction

Mobile gaming is a subset of video-gaming attracting players of all ages. In 2018 it was estimated that mobile gaming accrued approximately $\$ 68.5$ billion in revenue globally (Wijman, 2019). Of interest to game designers, and to those concerned about addictive behaviors, is the deep sense of flow during play. Flow has been described as an enjoyable state of absorption characterized by a distortion of time, and deep effortless concentration in any rewarding activity (Csikszentmihályi, 1990). Flow is known to increase one's desire to reengage in the situation that led to this pleasurable state (Keller, Ringelhan, \& Blomann, 2011). Flow, however, has a dark side. It has been implicated in the development of behavioral addictions such as gambling (Dixon et al., 2018), as well as video-game play (Keller, Ringelhan et al., 2011). Despite flow's prominence in the behavioral addiction literature, research surrounding the skill-challenge balance (a key antecedent of flow) is not well understood in the context of mobile gaming. Additionally, the motivational consequences of flow in mobile games remains relatively understudied. The current study seeks to investigate the relation between the skill-challenge balance and flow and examine how flow impacts the urge to keep playing.

\section{THE SKILL-CHALLENGE BALANCE AND FLOW}

Although there are several antecedents related to the emergence of flow, the skill-challenge balance is of particular interest since it is a cornerstone of video-game design. In all successful video games, as the skill of players increase, so too does the difficulty of the game. The 
relation between the skill-challenge balance and optimal flow is classically understood by means of the "channel model" depicted in panel A of Fig. 1. The model posits that optimal flow is produced whenever the challenge of a game matches the skills of a player. Numerous studies have shown that when skill and challenge are approximately equal, flow is experienced (the diagonal "channel" in Fig. 1, Panel A; Harmat et al., 2015; Baumann, Lürig, \& Engeser, 2016; Engeser \& Reinberg, 2008; Keller \& Bless, 2008; Keller, Ringelhan et al., 2011; Keller, Bless, Blomann, \& Kleinböhl, 2011; Kennedy, Miele, \& Metcalfe, 2014; Tozman, Zhang, \& Vollmeyer, 2017). In the channel model, it does not matter at what level of skill and challenge players are at. Even for beginners with little skill, so long as they are playing an easy game, flow should be experienced since their minimal skill is matched by the minimal challenge of the game. Moreover if flow is a highly motivating state (Keller \& Bless, 2008; Keller, Ringelhan et al., 2011), then even relatively novice players should be motivated to keep playing right from the introduction to a new game.

An alternative to the classic model of flow is what can be described as a quadrant model of flow (see Fig. 1, panel B). ${ }^{1}$ In this model, flow is experienced only when players feel that they have reached a high level of skill (offset by a high level of challenge). The quadrant model posits that the balance between challenge and skill does not always lead to optimal flow (Abuhamdeh \& Csikszentmihalyi, 2012; Jin, 2012; Løvoll \& Vittersø, 2014; Moneta \& Csikszentmihalyi, 1996). For players who feel they have minimal skill and are playing what they feel is a minimally challenging game, apathy rather than flow should ensue.

Some aspects of particular games would appear to support a quadrant model over the classic model. In games such as poker (a gambling game with a skill component), some contend that it is the skilled players who "might have a better chance to enter the life-affirming flow state" (Palomäki \& Salmela, p. 112, 2016). Such contentions could apply in any gaming situation in which skill improves with experience. For online videogame players, Liu (2017) suggests that "if players believe they have high skill levels and are capable of performing certain actions, they may be more likely to experience flow in the online game environment" (p. 154). Players who have played more frequently to develop their skill are at a point where they can take on higher game demands confidently. These players would fall in the "optimal flow" quadrant of the model. By contrast, novice players may not enter flow as easily as they are focusing on building fundamental skills and becoming familiar with a game environment by playing minimally challenging (yet balanced) levels. Novices would thus land in the "apathy" quadrant.

Empirical support for the notion that flow within video games is engendered by the balance of challenge and skill

${ }^{1}$ For the purposes of the current research, we are contrasting a classic model of flow and a more recent extension. Although there is a tertiary model known as the "eight channel model" of flow (Nakamura et al., 2012), the classic and quadrant model of flow appeared to be the most applicable to video-games, and indeed have been informed by research using video games to test their assumptions. comes from studies which manipulate how challenging the game is by increasing or decreasing the speed at which players must play (Harmat et al., 2015; Baumann et al., 2016; Engeser \& Reinberg, 2008; Keller \& Bless, 2008; Keller, Ringelhan et al., 2011; Keller, Bless et al., 2011; Kennedy et al., 2014; Tozman et al., 2017; Jin, 2012). For example, consider a skilled player, playing the classic arcade game Tetris in which players must rotate variously shaped blocks so that they form solid, gapless rows. The game can be made too easy by having the shapes fall very slowly, or too hard by having the shapes fall so quickly that even experienced players cannot rotate and align shapes into their optimal winning positions. When speed of play is manipulated, an inverted- $U$ relation between the perceived skill-challenge balance and flow is produced (Harmat et al., 2015; Baumann et al., 2016; Engeser \& Reinberg, 2008; Keller \& Bless, 2008; Keller, Ringelhan et al., 2011; Keller, Bless et al., 2011; Kennedy et al., 2014; Tozman et al., 2017; Vuorre \& Metcalfe, 2016). Specifically, optimal flow is produced when the speed-based demands matches the player's skill, and less flow ensues when skills far exceed demands (e.g., slow-paced and too easy). Similarly, less flow also ensues if the demands far exceed skills (e.g., far too fast-paced and hence too difficult). These relations are depicted in Fig. 1 Panel C. A heightened desire to re-engage in the activity appears to only be experienced at the apex of the inverted $U$ flow function (Keller, Ringelhan et al., 2011). That is the urge to keep playing appears to be maximized by flow.

In both the classic and quadrant models of flow, there are clear predictions concerning boredom. Both models posit that for games that players feel are far too easy, boredom should ensue. For games that are too hard "anxiety" should ensue (Peifer, Schulz, Schächinger, Baumann, \& Antoni, 2014; Keller, Bless et al., 2011; Harmat et al., 2015). One problem with using the label anxiety as a descriptor involves its relation with arousal which is known to have motivational effects on game play. For example, for many types of video games those playing at their current level may show arousal/anxiety as they become anxious about whether or not they will level-up. Previous research (Larche, Musielak, \& Dixon, 2017) also suggests that high arousal is associated with just failing to "level up" (a near miss outcome) - a finding which is more aligned with the balance of challenge and skill in the flow channel. Hence, while the predictions concerning boredom are relatively straightforward, the different sources of arousal may complicate predictions concerning the "anxiety" quadrants. We prefer the term "frustration" instead of anxiety in depictions of the models. Specifically, when challenge far exceeds skill, frustration ensues (Jin, 2011; Nakamura, Tse, \& Shankland, 2012). For frustration, the predictions are clear. For games that are too easy - frustration should be minimal, and for games that are balanced, there may be mild frustration from not leveling up. For games that are obviously too hard, frustration should be high.

In developing both the classic and quadrant models, in order to demonstrate the skill-challenge balance and its relation to flow the research method of choice was to vary 
A) Classic Model of Flow

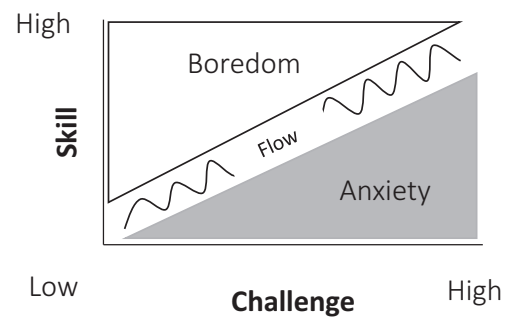

B) Quadrant Model of Flow

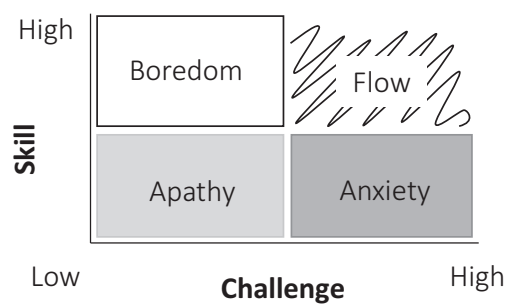

C) Skill-Challenge Balance Models "Inverted-U" Pattern of Flow

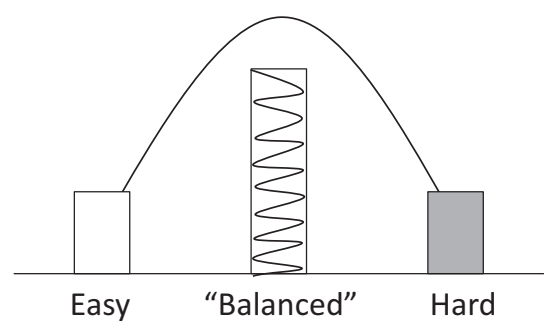

Fig. 1. Models of Flow based on the skill-balance. (A) Classic Model of Flow showing optimal flow emerges with a matching of challenge and skill (Csikszentmihályi, 1975) (B) Quadrant Model of Flow showing optimal flow emerging with high skill and high challenge (Csikszentmihalyi \& Csikszentmihalyi, 1992) (C) Inverted-U pattern of flow as posited by the skill-challenge balance models

the difficulty of games by varying the speed of play (slow play $=$ too easy, moderate speed $=$ balanced, very fast speeds = too hard; Harmat et al., 2015; Baumann et al., 2016; Engeser \& Reinberg, 2008; Keller \& Bless, 2008; Keller, Ringelhan et al., 2011; Keller, Bless et al., 2011; Kennedy et al., 2014; Tozman et al., 2017; Jin, 2012). Of course, increasing speed is not the only way to make games more difficult. Some games characterize difficulty based on the level of decision-making complexity. Consider for example the immensely popular puzzle game Candy-Crush. Speed of play is irrelevant. Rather, in each level of the game, the player must achieve a specific objective within a limited number of moves before the player can unlock the next level. The objectives can include bringing a certain number of 'ingredient' symbols to the bottom of the game matrix (in game play players are instructed to "Collect all 6 ingredients!"), or 'freeing' candy symbols encased in 'jelly' tiles (e.g., "Clear all the jellies!”). If the player makes the appropriate combination of moves to meet the objective within the allotted number of moves, they level up. This game uses what is called a ramping structure, such that as they level up, the puzzles become more complex and the game becomes progressively harder - in flow terminology, as player skills improve, the game elevates the challenge to maintain the optimal skill-challenge balance.

To date the skill-challenge balance has not been assessed in a mobile game like Candy-Crush in which challenge is manipulated by the complexity of the game. It is also unclear whether the experience of flow in a game like Candy-Crush occurs equally for those who feel unskilled, playing an easy game (i.e., where flow should still occur according to the classic model) or is more preferentially experienced by those who feel more highly skilled (the quadrant model).

\section{Overview of the current study}

The study has three aims. First, we aim to assess the relationship between the skill-challenge balance and flow when the skill-challenge balance is perturbed by game complexity rather than speed. The skill-challenge balance will be determined using an absolute difference score between perceived challenge and perceived skill. Here we seek to show that flow would be maximally induced by players attempting to play games at their current level standing (i.e., a balance of challenge and skill) whereas flow would be reduced when playing games that were either too hard or too easy. Games that are too easy are expected to be the most boring and least frustrating, while games that are too hard, should be the least boring yet most frustrating. Second, we seek to compare the classic and quadrant models of flow using players' subjective reports of skill, challenge and flow during gameplay. We expect to show that a game like Candy-Crush will more strongly support a quadrant model of flow in which the greatest flow would be experienced by those whose high perceived skill is balanced by high perceived challenge in the game (e.g., those falling into the hypothetical "flow" quadrant of the model), more so than those whose lower perceived skill is balanced by lower levels of challenge (e.g., those falling into the apathy quadrant of the model). Third we will examine whether the degree to which flow was experienced impacts the player's urge to continue playing. 


\section{METHODS}

\section{Participants}

A total of 72 participants were recruited from a pool of psychology students at the University of Waterloo. Eligible participants were those who: reached at least level 70 in the Candy-Crush Saga and had played within the last 12 months. Assigning a cut-off of level 70 in the Candy-Crush Saga ensured that the researchers could create a too-easy condition - a situation that would not be possible had we used more novice players. Level standings ranged from 77 to 828 in our sample.

The study's protocol was reviewed and approved by the University of Waterloo Research Ethics Committee. Consenting participants were advised that they could withdraw at any point in the study without penalty.

\section{Apparatus}

Lenovo tablets. Two Lenovo Tab 10" tablets were used one for gameplay and one to administer surveys using Qualtrics software.

Candy-Crush Saga game. Participants played a real version of the Candy-Crush Saga on a Lenovo Tablet device. The tablet device was mounted onto an inclined platform, facing a Logitech camera (1080 HDMI) that recorded the games.

\section{Materials}

Pre-test questionnaire. The pre-test questionnaire assessed demographic information (e.g., age, gender) and their general mobile gaming behavior (e.g., frequency of play).

Subjective arousal. Participants were asked to indicate their level of subjective arousal by pointing to a Self-Assessment Manikin (SAM; Lang, 1985) that best matched their in-game arousal level (see Fig. 2).

Urge-to-play. Two items were used to measure player urge (previously adapted from the Gambling Urge Scale; see Larche, Musielak, \& Dixon, 2017). Participants responded to "All I want to do is keep playing" and "I want to play so badly that I can almost feel it" using a 7-point Likert scale, with 1 indicating 'Strongly Disagree' and 7 indicating 'Strongly Agree'. The two items were summed. For multiple games of the same type (e.g., two easy games) these summed scores were averaged for each game condition.
Game Experience Questionnaire. The in-game module of the Game Experience Questionnaire (GEQ; IJsselsteijn, De Kort, \& Poels, 2013) was used to assess flow, perceived skill, perceived challenge and boredom during gameplay.

For flow, participants responded to the statements "I forgot everything around me" and "I felt completely absorbed". For skill, participants responded to the two items "I felt skilful" and "I felt successful". For challenge, participants responded to the two items "I felt challenged" and "I had put a lot of effort into it". Finally, for boredom participants responded to the single negative affect subscale item "I felt bored".

All items on the GEQ were answered on a 5-point scale, with 0 indicating 'not at all' and 4 indicating 'extremely'. Items for each construct (flow, challenge, skill and boredom) were summed then averaged (for games of the same type).

Skill-challenge balance. The skill challenge balance was defined as the absolute difference between perceived challenge ratings and perceived skill ratings respectively for each game (e.g., Skill-Challenge Balance $=\mid$ Challenge - Skill $\mid$ ).

Frustration. Subjective frustration was measured by having participants evaluate how much they agree/disagree with the statement "I feel frustrated" on a 7-point Likert scale, with 1 representing 'strongly disagree', and 7 representing 'strongly agree'.

\section{Design and skill-challenge balance manipulation}

Participants played two very easy games, four regular games, and two very hard games (eight games total). Regular games consisted of games that were near their current level standing (within a ten-level interval above or below the participant's current level standing). Hard games, were 100 levels above the participant's current level standing and would contain some features unfamiliar to players. Easy games involved levels at the very beginning (e.g. any level under level 10 in the game typically contains goals that can be easily reached).

Games were separated into two blocks of four games (one easy, two regular and one hard game in each block) with the games within blocks counterbalanced.

\section{Procedure}

Participants consent was obtained and their current level standing verified. Participants played two practice games to familiarize themselves with the procedure and the post-game surveys administered at the end of each game (e.g., including questions of boredom, flow, skill, challenge, positive affect, arousal, frustration, and urge-to-play). Participants then
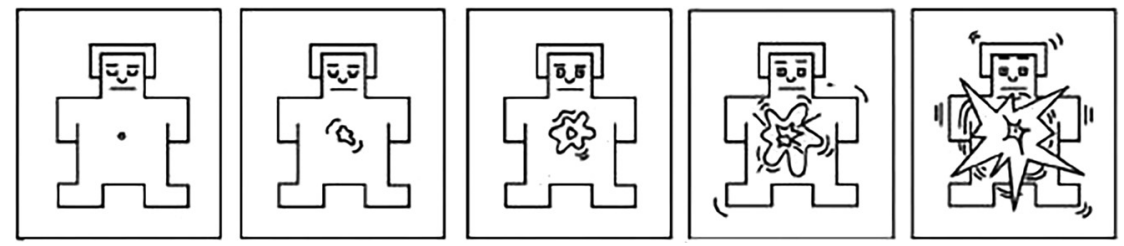

Fig. 2. Self-assessment manikins (SAMs) indexing subjective arousal from low arousal on the left, to high arousal on the right 

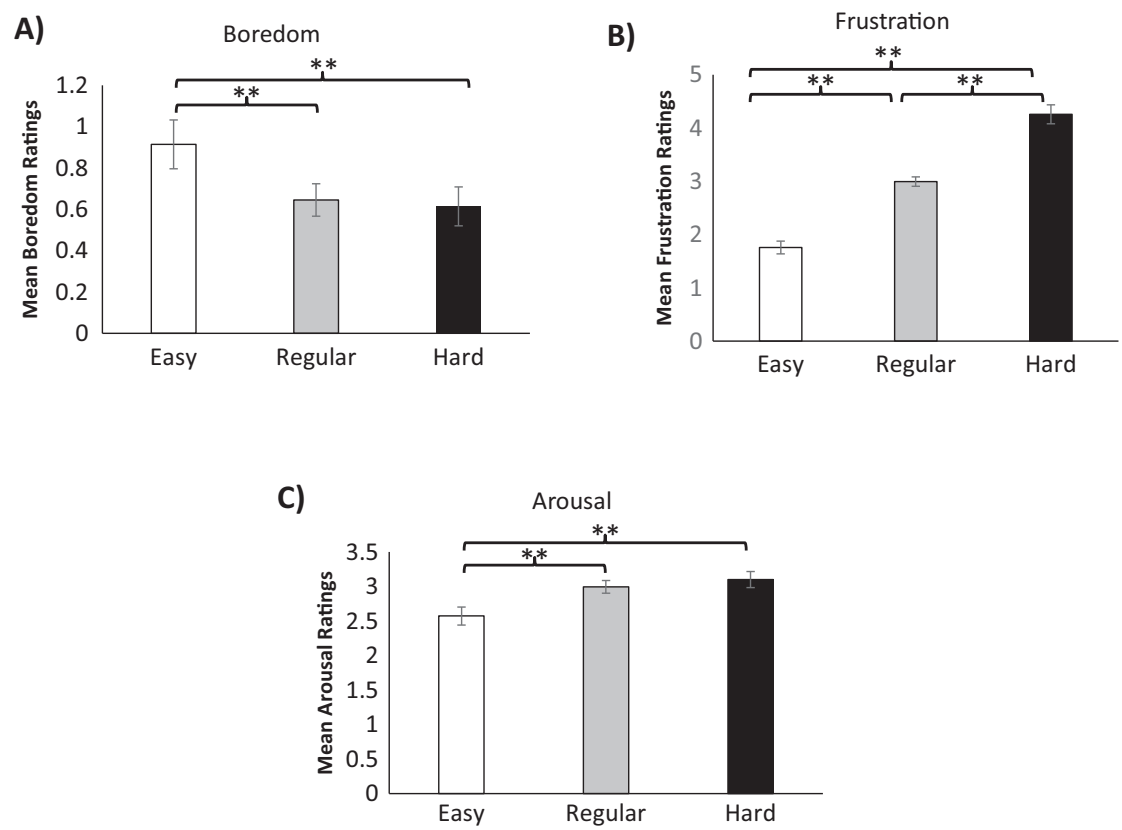

Fig. 3. Average (A) boredom, (B) frustration and (C) arousal scores across the three levels of difficulty. ${ }^{\star} P<0.01 .{ }^{*} P<0.05$

played the first block, followed by a second block of games (45 minutes of gameplay).

\section{Data analysis strategy}

Average scores for each dependent variable (e.g., boredom, frustration, arousal, skill, challenge, skill-challenge balance, flow, and urge) were calculated for each game type (easy, regular games and hard games) for each block of games. Since there were no statistical differences between block 1 and block 2 for each game type ( $P s>0.05$ for all measures) a single average for easy games, regular games and hard games was calculated for each participant.

Data points falling $3 \mathrm{SD}$ above or below the mean were considered outliers and removed. Normality was assessed for each measure using Kurtosis Fisher coefficients. Kurtosis statistics for each of our dependent variables fell within acceptable cut-off ranges (e.g., -1.96 and +1.96 ; see Gravetter \& Wallnau, 2014) allowing parametric statistics for analysis.

A series of one-way Repeated Measures ANOVAs assessed the influence of game type for each of the measures. Where violations of sphericity occurred Greenhouse Geisser corrections were applied to the degrees of freedom. Fisher's LSD post-hoc comparisons were used in the event of significant main effects.

\section{Ethics}

The study procedures were carried out in accordance with the Declaration of Helsinki. The Institutional Review Board of the University of Waterloo approved the study. All subjects were informed about the study and all provided informed consent.

\section{RESULTS}

Out of the 72 participants recruited, 12 had to be removed due to a failure of the difficulty manipulation. Specifically, we eliminated those who won a game that was supposed to be too hard, or those who lost an easy game. For the remaining 60 participants, their performance in the extreme games conformed to the difficulty manipulation - they all lost the games that were supposed to be too hard, and won the games designed to be too easy. For the regular condition, games were averaged regardless of outcome. Games at a player's level standing were assumed to be designed to compensate for skill level by presenting players with games whose complexity would be slightly greater than their skill level. Such a design strategy would not only ensure a balance between skill and challenge, but also create a variable-ratio reinforcement schedule, with winning outcomes being relatively infrequent and unpredictable. On average, players won only 1.12 games out of the 4 regular games they played.

We first looked at the experience of boredom, and frustration across each game difficulty condition to confirm whether these experiences conformed to the expected states hypothesized by both the classic models and the quadrant model (see Fig. 1, panels A and B where the "anxiety" area is instead denoted by "frustration"). We also measured arousal which previous research suggested might be high in both the regular game (due to excitement) and the too-hard game (due to frustration). Participants average experiences of boredom, frustration and arousal are shown in panels A, B and $\mathrm{C}$ of Fig. 3. Boredom scores statistically differed across the three conditions of difficulty, $F(1.44,85.02)=12.63, P<$ $0.001, \eta_{p}{ }^{2}=0.176$. Post-hoc comparisons showed that players 
A)

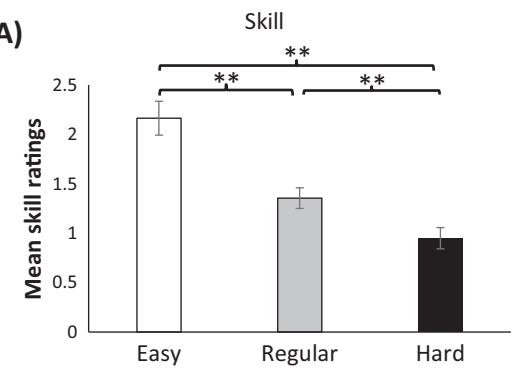

B)

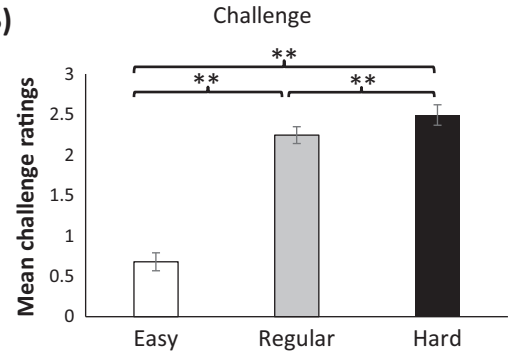

C)

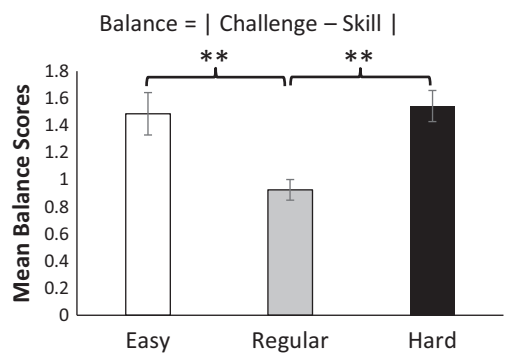

Fig. 4. (A) Subjective reports of skill across each game difficulty condition, (B) Subjective reports of challenge across each game difficulty condition, (C) the perception of balance, calculated as the absolute difference between subjective skill and challenge scores, with lower scores indicating greater balance. ${ }^{\star} P<0.01 .{ }^{\star} P<0.05$

felt the most bored while playing the easy games $(M=0.99$, $S D=0.89)$, and were less bored during the regular games $(M=0.64, S D=0.59)$ and the hard games $(M=0.58, S D=$ 0.70 ; all $P$ s $<0.001)$. There were no differences in boredom between the regular and hard games $(P=0.259)$.

Frustration also statistically differed across the three levels of difficulty, $F(2,118)=134.57, P<0.001, \eta_{p}{ }^{2}=$ 0.695. Post-hoc comparisons revealed players to be least frustrated during an easy game $(M=1.73, S D=0.927)$, slightly more frustrated during the regular games $(M=3.65$, $S D=1.26)$, and the most frustrated while playing the hard games $(M=4.27, S D=1.37$; all $P$ s $<0.001)$.

For arousal there was a significant main effect of difficulty level, $F(1.50,89.04)=15.62, P<0.001, \eta_{p}{ }^{2}=0.209$. Post-hoc comparisons indicated that the easy games $(M=$ $2.40, S D=0.98)$ induced lower arousal than the regular or hard games (both $\left.P_{\mathrm{s}}<0.001\right)$. The regular $(M=2.85, S D=$ $0.68)$ and hard games $(M=2.98, S D=0.88)$ did not differ $(P=0.108)$.

\section{Perceived skill, challenge, and the skill-challenge balance}

As a manipulation check confirming that our difficulty manipulation was capable of perturbing the skill-challenge balance, we assessed subjective ratings of challenge, skill, and balance respectively. Fig. $4 \mathrm{~A}$ and $\mathrm{B}$ show the means for skill and challenge across each level of difficulty. For skill there was a significant main effect of difficulty level, $F(1.41,83.54)$ $=55.53, P<0.001, \eta_{p}{ }^{2}=0.485$. Post-hoc comparisons indicated that players felt the most skilled while playing the easy games $(M=2.18, S D=1.21)$, slightly less skilled during the regular games $(M=1.39, S D=0.74)$, and the least skilled during the hard games $(M=0.96, S D=0.78$; all $P$ s $<0.001$; see Fig. $4 \mathrm{~A})$. For challenge scores there was a significant main effect of difficulty level, $F(1.46,86.21)=$ 142.94, $P<0.001, \eta_{p}{ }^{2}=0.708$. Post-hoc comparisons showed that players felt the easy games were the least challenging $(M=0.71, S D=0.81)$, the regular games slightly more challenging $(M=2.18, S D=0.75)$, and the hard games were the most challenging $(M=2.46, S D=$ 0.91; all Ps $<0.001$; see Fig. $4 \mathrm{~B})$.

Our metric of skill-challenge balance was calculated as the absolute difference of participants' respective skill and challenge ratings (shown in Fig. 4C). Using this metric there was a significant main effect of balance, $F(1.71,101.14)=$ 11.57, $P<0.001, \eta_{p}{ }^{2}=0.164$. That is, players felt little balance when playing the easy games $(M=1.47, S D=1.16)$, significantly more balance playing the regular games $(M=$ $0.91, S D=0.60 ; P<0.001)$ and significantly less balance playing the hard games $(M=1.54, S D=0.82 ; P<0.001)$. Balance for the hard and easy games did not statistically differ $(P=0.684)$.

\section{Skill-challenge balance and flow}

As shown in Fig. 5, our measures of flow depended on the difficulty of the game played. Specifically there was main effect of game difficulty, $F(1.65,97.37)=5.82, P=0.007$, $\eta_{p}{ }^{2}=0.09$. Post-hoc comparisons revealed that players experienced less flow for the easy games $(M=2.62, S D=$ $1.11 ; P=0.006)$ compared to the regular games $(M=2.83$, $S D=1.06 ; P<0.001)$ and the hard games $(M=2.83$, $S D=1.10 ; P=0.014)$. Intriguingly, regular and hard games were equally flow inducing $(P=0.922)$, even though players felt the harder games were too challenging for their level of 


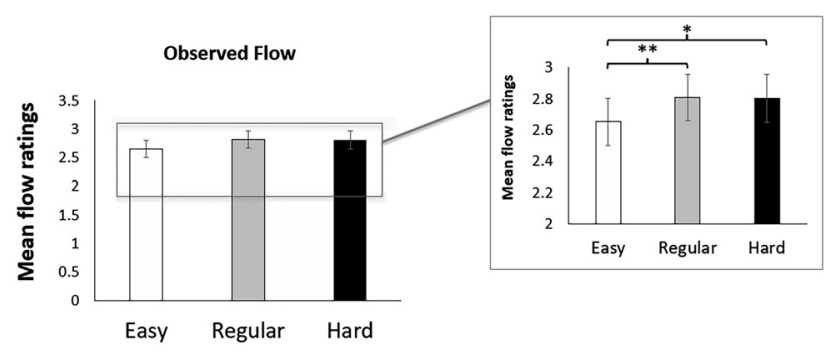

Fig. 5. Observed levels of flow across game difficulty conditions based on complexity (with subpanel highlighting effects with refined axis). ${ }^{\star} P<0.05,{ }^{\star \star} P<0.01$

skill (i.e., an imbalance of challenge and skill). Such results deviate from previous research which manipulated challenge via speed of play. It suggests that for games whose difficulty depends on complexity, games that are too easy will induce minimal flow, but even games that should be too difficult can still induce as much flow as games in which the skill challenge balance is optimized.

An axiom in game design is that as players get better the designers compensate by making the game harder (i.e., they maintain the skill-challenge balance). This can be demonstrated by assessing the correlation between perceived skill and challenge for games near their current play level (i.e., the regular games). Specifically, if game designers did not adjust challenge to meet players skill then there would be no correlation between challenge and skill - however, if they compensated for increasing skill by increasing challenge then a significant correlation would be expected. Fig. 6 shows a scatterplot of player average ratings of skill and challenge following these regular games as well as a theoretical (orange) line depicting an exact match of challenge and skill as well as the (dashed) line of best fit. There was a significant correlation between skill and challenge for the regular games, $r(58)=0.478, P<0.001$. As shown in the figure most data points fall below the theoretical line. This

\section{Skill-Challenge Balance for Regular Games}

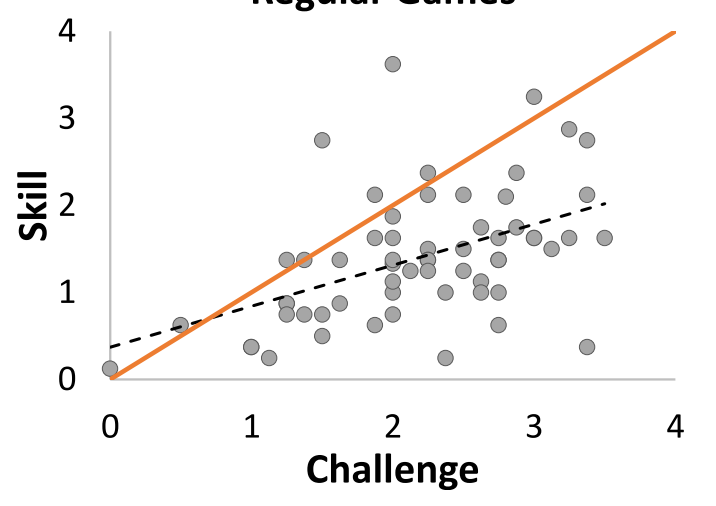

Fig. 6. Scatterplot depicting the relation between skill and challenge for all participants for the regular games. Orange line indicates the theoretical slope of 1 indexing a skill-challenge balance. (For interpretation of the references to color in this figure legend, the reader is referred to the web version of this article.) reflects developers compensating for a players' high skill by making the game slightly harder than their skill level. This makes sense when one considers how outside of the laboratory players achieved their current level. They may have had played 10 games and lost them all, then leveled up on the 11th game. If the game difficulty increases with the new level achieved, it would maintain a situation where challenge slightly, but consistently, exceeds skill.

To assess whether flow in Candy-Crush adhered more to the quadrant model of flow than the classic model we separated participants into the upper and lower quartiles of flow. Here we analyzed only the regular games since they induced both greater skill-challenge balance and flow. Table 1 displays the means and standard deviations of the upper and lower quartiles of flow for these games, $t(28)=$ $-16.33, P<0.001$.

Fig. 7 displays scatterplots representing skill, challenge and flow quartile. The white dots represent those in the lower quartile of flow scores, black dots those in the upper quartile.

In Fig. 7, the theoretical slope for a perfect 1:1 ratio of skill and challenge scores is displayed for reference. This line reflects a perfect match of challenge and skill, where we would expect the highest flow scores to emerge. For the regular games, one can see four scores falling directly on, or adjacent (touching) this theoretical line - all four of these participants were in the lower quartile of flow scores. Hence, the classic model does not adequately capture flow induction as predicted by the perfect matching of challenge and skill.

In Fig. 7 one sees the ramification of increasing the difficulty of the game - the majority of points fall further below the theoretical line depicting a perfect balance of skill and challenge. Fig. 8 shows the upper and lower quartile scorers for the regular games. In this figure we have drawn the key quadrants of interest (the "apathy" quadrant in the lower left, and the "flow" quadrant in the upper right). Visually one can see that far more high flow scores fall into the high skill and high challenge quadrant for the regular games condition (six out of the 15 upper quartile scores, versus zero out of 15 lower quartile scores). ${ }^{2}$ By contrast, the majority of low flow scores fall into the low skill and low challenge quadrant of the graph (eight out of 15 lower quartile scores), with only one high flow score in this quadrant. A chi-square test of independence confirmed that whether a player experienced high or low flow in the regular games depended on which of the two key quadrants players

Table 1. Means of upper and lower flow quartiles for regular games

\begin{tabular}{lccc}
\hline & \multicolumn{3}{c}{ Regular Games } \\
\cline { 2 - 4 } & $n$ & Flow & SD \\
\hline Total lower quartile & 15 & 0.488 & 0.33 \\
Total upper quartile & 15 & 3.16 & 0.50 \\
\hline
\end{tabular}

${ }^{2}$ For this particular analysis we only considered scores that were unambiguously falling into the key quadrants of interest (e.g., flow and apathy). 


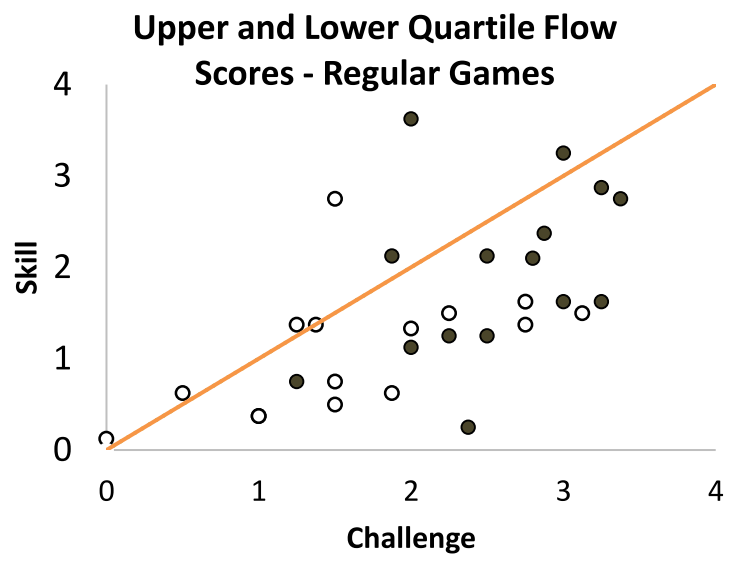

Fig. 7. Scatterplot representing the skill-challenge balance for the upper and lower quartiles of flow experienced during the regular games. White dots represent the lower quartile of flow scores while black dots represent the upper quartile of flow scores. Orange line indicates the theoretical slope of 1 indexing a skill-challenge balance. (For interpretation of the references to color in this figure legend, the reader is referred to the web version of this article.)

landed. Players in the upper quartile of flow were significantly more likely to be in the upper quadrant and players in the lower quartile of flow were significantly more likely to be in the lower quadrant, $\chi^{2}(1)=10.81, P<0.05$.

One problem with the above quadrant analysis is when players in the regular game are playing near their current level, it is likely that challenge will be slightly higher than their skill level for most of the games that they play. This causes problems in determining exactly where the "quadrants" should lie. According to the quadrant model, however, the general principle is that those with higher perceived challenge and skill will be more likely to experience flow than those with lower perceived challenge and skill. If so, then those in the upper quartile of flow should have significantly higher challenge, and significantly higher skill scores than those in the lower quartile of flow scores. Also if one were to combine challenge and skill scores into a single metric (challenge plus skill scores) those in the upper quartile of flow scores should have higher combined scores than those in the lower quartile. Table 2 shows that these contentions are all statistically true for the regular games.

The same relation between absolute levels of challenge, skill and flow holds when considering the entire sample. For the regular games the combined scores (challenge plus skill) are significantly positively correlated with flow scores, $r(58)=0.597, P<0.001$.

\section{Flow experiences and the urge-to-play}

Fig. 9 depicts the mean urge-to-play ratings across the different levels of difficulty. A repeated measures ANOVA revealed a significant main effect of difficulty on the urge to play, $F(1.63,96.71)=11.46, P<0.001, \eta_{p}^{2}=0.163$. Posthoc comparisons revealed significantly less urge induced by the easy games $(M=6.84, S D=3.08)$ compared to the regular games $(M=7.58, S D=2.94 ; P<0.001)$ and the

\section{Skill-Challenge Balance and Flow Scores - Regular Games}

(A)

(B)

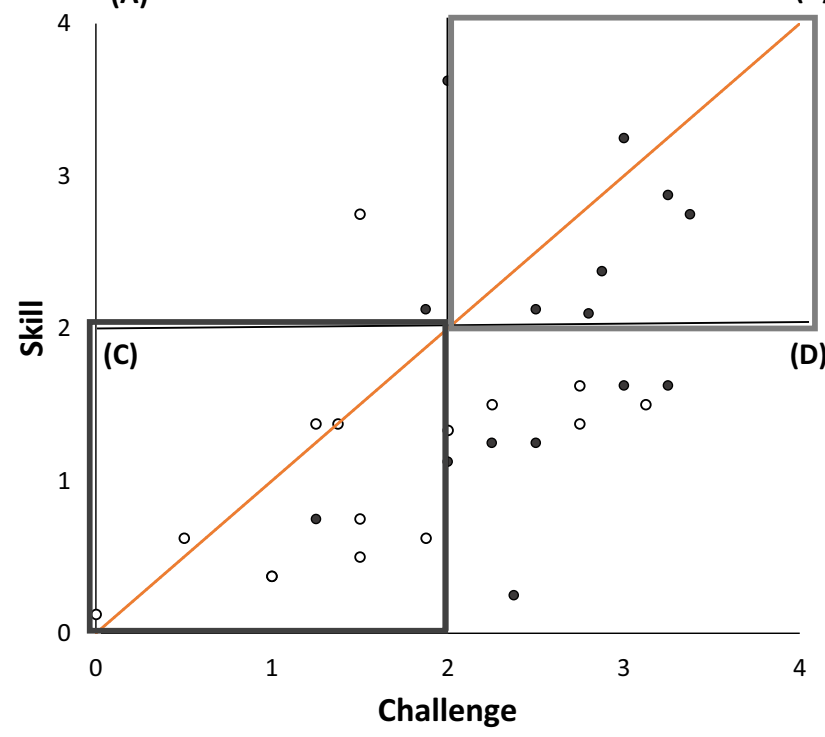

Fig. 8. Scatterplot depicting the relation between skill, challenge and flow, highlighting the lower quartile (white dots) and upper

quartile (black dots) of flow scores for the regular games. In assessing the "quadrant model" of flow, the gray box on the graph indicates the high skill and high challenge quadrant containing the preponderance of high flow scores, while the black box indicates the preponderance of low flow scores in the low skill and low challenge quadrant. Orange line represents theoretical slope of 1 indexing the skill-challenge balance. (For interpretation of the references to color in this figure legend, the reader is referred to the web version of this article.)

Table 2. Independent $t$-tests for the means (and standard deviations) of skill, challenge, and the means of skill + challenge between those in the upper and lower quartiles of flow for the regular games

\begin{tabular}{lccccc}
\hline & $n$ & Challenge & SD & $t$ & $P$-value \\
\hline $\begin{array}{c}\text { Lower quartile } \\
\text { of flow }\end{array}$ & 15 & 1.62 & 0.85 & -3.42 & 0.002 \\
$\begin{array}{c}\text { Upper quartile } \\
\text { of flow }\end{array}$ & 15 & 2.55 & 0.60 & & \\
\hline $\begin{array}{c}\text { Lower quartile } \\
\text { of flow }\end{array}$ & 15 & 1.08 & 0.68 & -2.85 & 0.008 \\
$\begin{array}{c}\text { Upper quartile } \\
\text { of flow }\end{array}$ & 15 & 1.94 & 0.94 & & \\
$\begin{array}{c}\text { Lower quartile } \\
\text { of flow }\end{array}$ & 15 & 2.70 & 1.35 & -3.67 & 0.001 \\
$\begin{array}{c}\text { Upper quartile } \\
\text { of flow }\end{array}$ & 15 & 4.49 & 1.30 & & \\
\hline
\end{tabular}

N.B. $P$-values are statistically significant at $a=0.05$ 


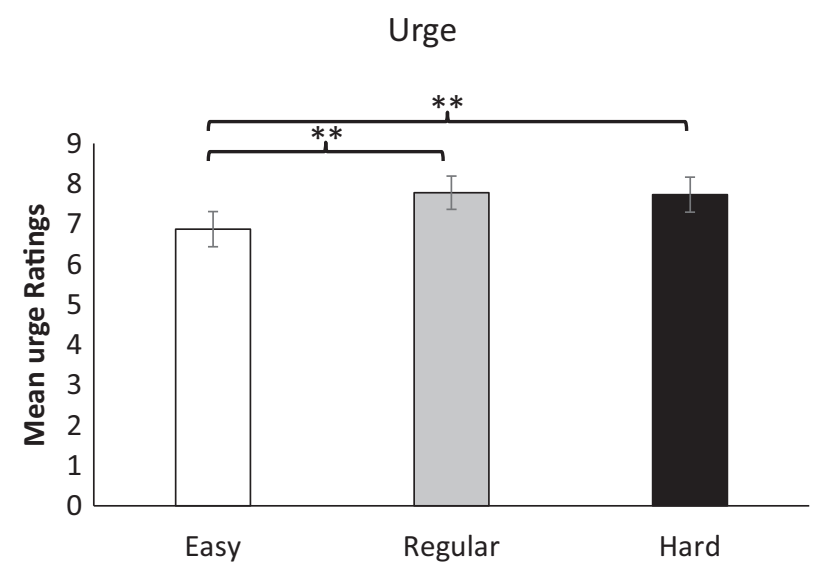

Figure 9. Average urge ratings across the three levels of difficulty. ${ }^{\star} P<0.01 .{ }^{*} P<0.05$

hard games $(M=7.70, S D=3.13 ; P<0.001)$. Similar to our results for arousal and flow, while easy games were minimally urge inducing, regular and hard games were equally effective in inducing the urge to continue playing $(P=$ 0.413).

Our converging patterns of arousal and flow make it difficult to tease apart whether flow has a unique influence on urge, over and above arousal (which is known to influence urge in Candy-Crush; see Larche et al., 2017). To address this, we used hierarchical regression to determine the extent to which flow drives urge independent of changes in arousal. We restricted our analyses to the regular and hard games conditions since both flow and urge were substantially greater in these conditions. Since the relationship between arousal and urge is well established in the gaming literature, arousal was therefore entered at Step 1 of our model predicting urge. Flow was then entered at Step 2 of the model. At Step 1, arousal accounted for $44.2 \%$ of unique urge variance, $F(1,58)=45.86, P<0.001$. Flow then accounted for an additional $21.8 \%$ of unique urge variance over and above arousal, $R^{2}=0.660, F$-change $(1,57)=$ $36.69, P<0.001$. Similarly for the hard games, at Step 1 arousal accounted for $46.5 \%$ unique urge variance, $F(1,58)$ $=50.46, P<0.001$. At Step 2 flow accounted for an additional $20.2 \%$ unique urge variance over and above arousal, $R^{2}=0.667$, F-change $(1,57)=34.4, P<0.001$.

\section{DISCUSSION}

The current study assessed the relationship between the skill-challenge balance and flow in a mobile video-game as well as the affective consequences that accompany flow (e.g., boredom, frustration, and arousal). As in previous research we perturbed the skill-challenge balance by manipulating difficulty. In Candy-Crush, however, game difficulty was manipulated by the complexity of the moves required to solve the puzzle rather than the speed manipulations that have been used in previous research. We also compared two models of flow, namely the classic channel model and the quadrant model of flow - each carrying very different implications of optimal flow for players of different perceived skill levels. We demonstrated that those with low perceived skill experienced low flow even if there was an exact match between their low perceived skill and low perceived challenge - a data pattern that contradicts the classic channel model. Conclusively we showed that those with high perceived skill experienced greater flow than those with low perceived skill - a data pattern consistent with the quadrant model. We also showed that flow is uniquely and highly motivating in an authentic game setting.

Firstly, levels of boredom and frustration across the different levels of difficulty mirrored predictions of the classic and quadrant models of flow. Easy games were the most boring as players were able to level up with ease. As difficulty increased games became less boring, such that the regular and hard games were less boring than the easy games. Interestingly, scores for boredom were relatively low across all levels of difficulty (scores less than 1), attesting to the appealing nature of Candy-Crush as a game in general. Harder games were also the most frustrating as players could not reach the game's objectives, with regular games being less frustrating, and the easy games the least frustrating. Because players were playing on a designated device rather than their own, we would perhaps expect these frustration scores to be amplified in more naturalistic settings.

Our findings for arousal highlight difficulties in labeling the too-hard games as anxiety provoking. Easy games were the least arousing to play compared to the regular games. However, hard games were as arousing as regular games. Specifically, arousal can increase in balanced games due to the excitement and proximity to leveling up, and arousal can also increase in too hard games due to frustration. In Panels A and B of Fig. 1 situations in which challenge far outweighs skill are labeled using the term anxiety. We would argue that players may also become anxious even in the flow channels just as arousal is heightened due to the proximity of leveling up, so too may anxiety heighten since players during these balanced games cannot be sure whether they will succeed or fail. We would suggest that a more appropriate label for situations in which challenge clearly outweighs skill is frustration. Here we show that frustration conforms to all perturbations of the skill challenge balance - it is low in easy games, higher in regular games and highest in games that are too hard. Although we did not explicitly measure anxiety given the ambiguous results we show with arousal, frustration may be a superior concept to demarcate situations where challenge far outweighs skill.

Our skill-challenge balance manipulation using difficulty manipulations in Candy-Crush successfully replicated what has been previously observed by past research (Harmat et al., 2015; Baumann et al., 2016; Engeser \& Reinberg, 2008; Keller \& Bless, 2008; Keller, Ringelhan et al., 2011; Keller, Bless et al., 2011; Kennedy et al. 2014; Tozman et al., 2017; Vuorre \& Metcalfe, 2016). Players felt the most balanced when playing games around their level standing, and the least balanced when playing levels that were too easy (i.e., 
skill far outweighing challenges in the game) or levels that were far too hard (i.e., challenges far outweighing skill).

Despite successfully perturbing the balance of challenge and skill our results only partially reflected the inverted-U shape pattern of flow shown in previous research. Consistent with prior research players experienced the least flow during the very easy games, and substantially more flow during the regular games by comparison. However, the hard games triggered comparable flow to the regular games, suggesting that players were not overwhelmed by the higher challenge. One reason for this may be because highly challenging complex games may not disrupt players' sense of control or agency during play (Chen, 2007; Vuorre \& Metcalfe, 2016). In speed-based games like Tetris, a player must react reflexively to increasing demands in order to succeed. If a speed-based game is too fast, players may not be able to interact with the game at all, and this may thwart flow. In Candy-Crush players are not bound to any time constraint, and thus can still interact with the hard games.

A defining feature of flow is that it is a state of deep, effortless concentration (Csikszentmihályi, 1990). The low flow observed among novices could be explained by lack of necessary skills to feel that they were able to progress effortlessly through gameplay, and thus they may have a more difficult time entering flow compared to advanced players (Wu, Scott, \& Yang, 2013). However, this explanation warrants further research.

Crucially, in line with hypotheses facilitated by Keller, Ringelhan et al. (2011), players reported higher urge-to-play following the regular and hard games, where flow was experienced, and relatively less urge-to-play following the easy games where flow was curtailed. The hierarchical regression demonstrated that flow contributed unique urge variance over and above arousal - a primary reinforcer of gaming behavior (Anderson \& Brown, 1984; Poels, Hoogen, Ijsselsteijn, \& de Kort, 2012). Combined with our quadrant analysis, the increased flow observed among those with higher perceived skill players suggests these players are at a higher risk for problems given the enjoyable and motivating state of flow (Kubey \& Csikszentmihalyi, 2002; Wu et al., 2013). Such high flow may in part account for why problem players report playing for longer than intended, since it may be more difficult for these players to come out of gameplay with ease. This in itself may increase the potential for problems associated with excessive gameplay, especially if playing longer and more frequently interferes with other aspects of daily life (Hull, Williams, \& Griffiths, 2013).

\section{Limitations}

Although we had players indicate their intentions to continue gameplay, whether or not this translates to behavioral persistence remains to be explored. In a similar vein, CandyCrush is a mobile game that features microtransactions (e.g., opportunities to purchase or gain additional features or "power-ups" that may advance gameplay). This inherent aspect of gameplay was disabled in this current study in order to standardize play as much as possible between participants. Another limitation concerns the breadth of our measure of flow. As briefly aforementioned, flow is a complex construct with multiple antecedents and consequences, including time distortion, loss of self-awareness and actionawareness merging. Future studies might also consider measuring these other facets in a game setting to contribute to a comprehensive understanding of the mechanism of flow. Finally, since Candy-Crush is only one example of a game which varies difficulty via complexity rather than speed. Future research should aim to reproduce our results using other games of varying difficulty by varying complexity.

\section{CONCLUSION}

In conclusion, our findings provide a nuanced perspective of the relationship between the skill-challenge balance and flow as it emerges in games of complex difficulty. The classic skillchallenge balance model of flow may not capture experience in games with a complex difficulty structure since players experienced higher flow in both balanced and more challenging games. We also demonstrate the importance of expertise in the skill-challenge balance, since our findings suggest that more advanced players may be more susceptible to the deep, immersive experience of flow. Flow was also a highly invigorating experience in the context of mobile game play, which from a harm perspective suggests a heightened potential for problems in players experiencing higher degrees of immersion.

Funding sources: This research was supported by the Natural Sciences and Engineering Research Council of Canada through research Grants to M.J.D.

Authors' contribution: The authors of the current paper include the following: Chanel J. Larche and Mike J. Dixon. CJL and MJD contributed to the theoretical development, data analysis and preparing the final manuscript. CJL collected the data for this project.

Conflict of interest: The authors declare that they have no conflict of interest.

\section{REFERENCES}

Abuhamdeh, S., \& Csikszentmihalyi, M. (2012). The importance of challenge for the enjoyment of intrinsically motivated, goaldirected activities. Personality and Social Psychology Bulletin, 38(3), 317-330.

Anderson, G., \& Brown, R. I. F. (1984). Real and laboratory gambling, sensation-seeking and arousal. British Journal of Psychology, 75(3), 401-410.

Baumann, N., Lürig, C., \& Engeser, S. (2016). Flow and enjoyment beyond skill-demand balance: The role of game pacing curves and personality. Motivation and Emotion, 40(4), 507-519. 
Chen, J. (2007). Flow in games (and everything else). Communications of the ACM, 50(4), 31-34.

Csikszentmihályi, M. (1990). The domain of creativity. In M. A. Runco \& R. S. Albert (Eds.), Sage focus editions, Theories of creativity (Vol. 115, pp. 190-212). Sage Publications, Inc.

Csikszentmihalyi, M., \& Csikszentmihalyi, I. S. (eds.). (1992). Optimal experience: Psychological studies of flow in consciousness. Cambridge University Press.

Csıkszentmihályi, M. (1975). Beyond boredom and anxiety. In Book beyond boredom and anxiety (1975 edn). San Francisco, CA: Jossey-Bass.

Dixon, M. J., Stange, M., Larche, C. J., Graydon, C., Fugelsang, J. A., \& Harrigan, K. A. (2018). Dark flow, depression and multiline slot machine play. Journal of Gambling Studies, 34(1), 73-84.

Engeser, S., \& Rheinberg, F. (2008). Flow, performance and moderators of challenge-skill balance. Motivation and Emotion, 32(3), 158-172.

Gravetter, F., \& Wallnau, L. (2014). Essentials of statistics for the behavioral sciences (8th ed.). Belmont, CA: Wadsworth.

Harmat, L., de Manzano, Ö., Theorell, T., Högman, L., Fischer, H., \& Ullén, F. (2015). Physiological correlates of the flow experience during computer game playing. International Journal of Psychophysiology, 97(1), 1-7.

Hull, D. C., Williams, G. A., \& Griffiths, M. D. (2013). Video game characteristics, happiness and flow as predictors of addiction among video game players: A pilot study. Journal of behavioral addictions, 2(3), 145-152.

IJsselsteijn, W. A., De Kort, Y. A. W., \& Poels, K. (2013). The game experience questionnaire (pp. 3-9), Eindhoven: Technische Universiteit Eindhoven.

Jin, S. A. A. (2011). "I feel present. Therefore, I experience flow:" A structural equation modeling approach to flow and presence in video games. Journal of Broadcasting \& Electronic Media, 55(1), 114-136.

Jin, S. A. A. (2012). “Toward integrative models of flow”: Effects of performance, skill, challenge, playfulness, and presence on flow in video games. Journal of Broadcasting \& Electronic Media, 56(2), 169-186.

Keller, J., \& Bless, H. (2008). Flow and regulatory compatibility: An experimental approach to the flow model of intrinsic motivation. Personality and Social Psychology Bulletin, 34(2), 196-209.

Keller, J., Bless, H., Blomann, F., \& Kleinböhl, D. (2011). Physiological aspects of flow experiences: Skills-demand-compatibility effects on heart rate variability and salivary cortisol. Journal of Experimental Social Psychology, 47(4), 849-852.

Keller, J., Ringelhan, S., \& Blomann, F. (2011). Does skills-demands compatibility result in intrinsic motivation? Experimental test of a basic notion proposed in the theory of flow-experiences. The Journal of Positive Psychology, 6(5), 408-417.

Kennedy, P., Miele, D. B., \& Metcalfe, J. (2014). The cognitive antecedents and motivational consequences of the feeling of being in the zone. Consciousness and Cognition, 30, 48-61.
Kubey, R., \& Csikszentmihalyi, M. (2002). Television addiction is no mere metaphor. Scientific American, 286(2), 74-80.

Lang, P. J. (1985). The cognitive psychophysiology of emotion: Anxiety and the anxiety disorders. Hillsdale, NJ: Lawrence Erlbaum.

Larche, C. J., Musielak, N., \& Dixon, M. J. (2017). The candy crush sweet tooth: How 'near-misses' in candy crush increase frustration, and the urge to continue gameplay. Journal of Gambling Studies, 33(2), 599-615.

Lemmens, J. S., Valkenburg, P. M., \& Peter, J. (2011). Psychosocial causes and consequences of pathological gaming. Computers in Human Behavior, 27(1), 144-152.

Liu, C. C. (2017). A model for exploring players flow experience in online games. Information Technology \& People.

Løvoll, H. S., \& Vittersø, J. (2014). Can balance be boring? A critique of the "challenges should match skills" hypotheses in flow theory. Social Indicators Research, 115(1), 117-136.

Moneta, G. B., \& Csikszentmihalyi, M. (1996). The effect of perceived challenges and skills on the quality of subjective experience. Journal of Personality, 64(2), 275-310.

Nakamura, J., Tse, D. C. K., \& Shankland, S. (2012). Flow: The experience of intrinsic motivation. In R. M. Ryan (Ed.). The Oxford handbook of human motivation (pp. 169-185). OUP USA.

Palomäki, M. L. Á. J., \& Salmela, M. (2016). Poker players with experience and skill are not "Ill": Exposing a discrepancy in measures of problem gambling. Journal of Gambling and Commercial Gaming Research, 1, 1-18.

Peifer, C., Schulz, A., Schächinger, H., Baumann, N., \& Antoni, C. H. (2014). The relation of flow-experience and physiological arousal under stress-can u shape it?. Journal of Experimental Social Psychology, 53, 62-69.

Poels, K., Hoogen, W. V. D., Ijsselsteijn, W., \& de Kort, Y. (2012). Pleasure to play, arousal to stay: The effect of player emotions on digital game preferences and playing time. Cyberpsychology, Behavior, and Social Networking, 15(1), 1-6.

Tozman, T., Zhang, Y. Y., \& Vollmeyer, R. (2017). Inverted Ushaped function between flow and cortisol release during chess play. Journal of Happiness Studies, 18(1), 247-268.

Vuorre, M., \& Metcalfe, J. (2016). The relation between the sense of agency and the experience of flow. Consciousness and Cognition, 43, 133-142.

Wijman, T. (2019, June 18th). The Global games market will generate $\$ 152.1$ Billion in 2019 as the U.S. overtakes China as the biggest market. NewZoo.

Wu, T. C., Scott, D., \& Yang, C. C. (2013). Advanced or addicted? Exploring the relationship of recreation specialization to flow experiences and online game addiction. Leisure Sciences, 35(3), 203-217.

\footnotetext{
Open Access. This is an open-access article distributed under the terms of the Creative Commons Attribution-NonCommercial 4.0 International License (https:// creativecommons.org/licenses/by-nc/4.0/), which permits unrestricted use, distribution, and reproduction in any medium for non-commercial purposes, provided the original author and source are credited, a link to the CC License is provided, and changes - if any - are indicated.
} 